\title{
Die Menschen ihre Geschichte erzählen lassen
}

\section{Adressaten als Kompetenzquellen in der Sozialen Arbeit}

Bernd Birgmeier

PD Dr. Bernd Birgmeier ist

Akademischer Rat am Lehrstuhl für Sozialpädagogik und

Gesundheitspädagogik an der

Katholischen Universität Eichstätt-

Ingolstadt.

E-Mail

bernd.birgmeier@ku-eichstaett.de
Die professionelle Soziale Arbeit muss die Autonomie der Lebenspraxis ibrer Adressaten achten. Dazu gehört, die Menschen selbst nach ibrer individuellen Geschichte $z u$ fragen und gemeinsam zu erkunden, wie es zu den Problemen bei ihrer Lebensführung und Lebensbewältigung gekommen ist.

Spätestens seit der Umstrukturierung der Ausbildung in Sozialer Arbeit im Zuge des Bologna-Prozesses scheinen Kompetenzen - zumindest für das erfolgreich abzuschließende Hochschulstudium - das Maß aller Dinge zu sein. Dabei ist meist die Rede von einer Reflexions-, Wissens-, Anwendungs- oder einer praktischen und methodischen Kompetenz, die es (gemessen an definierten Standards) zu erwerben gilt, um in den spezifischen Arbeitsfeldern auch professionell handeln zu können.

Die Vermittlung solcher Kompetenzen geschieht jedoch nicht analog, also quasi als eindimensionaler Transfer diverser Wissensbestände von » $\mathrm{A}$ nach $\mathrm{B}$ « oder gar nach dem Motto: Hier die Kompetenz - dort die Inkompetenz, sondern wie es der Begriff der Ver-Mittlung bereits erahnen lässt - zwischen den beteiligten Personen in Form eines diametrischen, dialogischen und - eben zwischenmenschlichen Prozesses auf der Basis zwischenmenschlicher Beziehungen.

Ein solches Grundverständnis zur Kompetenzvermittlung gilt in der Ausbildung, vor allem aber auch in der Praxis Sozialer Arbeit, die - besonders in ihrer Lesart als "personenbezogene Dienstleistung « (vgl. Effinger 2009) - im Regelfall nicht nur zwischen Personen geschieht, sondern konkret an bestimmte Personen adressiert ist. Der Adressat ist Ausgangspunkt, Partner und Ziel jeglicher professioneller Hilfe.

Und dies aus gutem Grund: denn die Sozialarbeiterin und der Sozialpädagoge kann erst dann planen, was er an Vermittlungs-Diensten leisten soll, wenn er bezogen auf die Bedarfe des Adressaten - weiß, was dieser (auch an Kompetenzen) braucht! Kompetenzdebatten in Bezug auf das professionelle Handeln erhalten deshalb erst dann ihre Legitimation, wenn sie sich auf die Bedarfe von Kompetenzen der Adressaten beziehen. Daher hat der Adressat immer im Zentrum aller kompetenzorientierten Denk- und Handlungslogiken der Sozi-alen Arbeit zu stehen.

\section{Kompensationskompetenzen}

Doch um welche Adressaten-Kompetenzen soll es nun konkret gehen, an denen sich die Soziale Arbeit als Disziplin und Profession - denkend und handelnd - orientieren soll? Vielleicht, ganz allgemein, an Kompetenzen, mit denen vermeintliche Inkompetenzen kompensiert werden können? Soziale Arbeit also überspitzt formuliert - als Zentralinstanz für die Vermittlung von »Inkompetenzkompensationskompetenzen « (vgl. Marquard 2005)?

Möglich! Doch es wäre - wie bereits angedeutet - höchst vermessen anzunehmen, die Soziale Arbeit legitimierte ihre Kompetenz alleinig dort, wo sie (hilfebedürftigen!) Personen hilft, ihre scheinbaren Inkompetenzen zu kompensieren. Eine solche Grundhaltung wäre nicht nur schon deshalb schlichtweg falsch, weil Kompetenz niemals einen vollendeten, abgeschlossenen Zustand beschreiben kann, sondern allenfalls ein generelles Prinzip und einen immerwährenden, reaktiven Prozess, der im Auftrag steht, mit den gegebenen Mitteln und Möglichkeiten etwaige Inkompetenzen stetig neu zu bewältigen.

Heute kompetent zu sein schließt demnach nicht aus, morgen inkompetent zu werden - zumal bis dato ohnehin keine Kriterien dafür zu ersehen sind, die exakt angeben, wo nun konkret die Kompetenz beginnt und die Inkompetenz aufhört!

Im Blick auf diese »Grauzone «, die sich zwangsweise zwischen den Phänomenen 
der Kompetenz und Inkompetenz offenbart, sind alle Organe, Institutionen und Personen, die für die Soziale Arbeit stehen und an die auch die Kompetenzdebatten geknüpft sind, weder als kompetent noch als inkompetent zu bezeichnen; vielmehr sind sie - bedingt durch den rasanten gesellschaftlichen Wandel und die Multidimensionalität sozialer Probleme - in einem stetigen Prozess des »Dazwischen « (d. h. zwischen Bewährtem und Neuem) befangen, den es vor allem durch Lernprozesse zu kompensieren gilt.
Aus der Perspektive des Adressaten geht es daher um Kompetenzen für das Leben, das sowohl allgemeine Existenzialkompetenzen als auch spezifische Residualkompetenzen einfordert. Entscheidend für diese Differenzierung ist einmal die Tatsache, dass jeder Mensch grundsätzliche, existenzielle Kompetenzen benötigt, um Person zu werden; pädagogische Prinzipien, wie Mündigkeit, Selbsttätigkeit und Selbstbestimmung spielen hierbei eine wichtige Rolle. Andererseits werden solche Existenziale (oder: Le-

\section{"Was ist wichtiger: die gute Soziale Arbeit oder das gelingende Leben des Adressaten? "}

Je schwieriger es ist, klare Abgrenzungsmerkmale zwischen Kompetenz und Inkompetenz zu bestimmen, umso notwendiger erscheint eine Festlegung nach der Relevanz und Wichtigkeit diverser Kompetenzen im Kontext ihrer Zielabsichten und Funktionsbereiche. Wofür sind überhaupt Kompetenzen nötig und welche Kompetenzen sind wichtig? Um was soll es gehen: um reine berufsorientierte Kompetenzen, die der Sozialarbeiter oder Sozialpädagoge braucht oder um lebensorientierte Kompetenzen, die der Adressat zum Leben seines Lebens benötigt? Was ist wichtiger: die (gute) Soziale Arbeit oder das (lebenswerte, gelingende, gute) Leben des Adressaten?

\section{Lebensbewältigungs- und Lebensführungskompetenzen}

Abgesehen davon, dass es für beide Perspektiven wichtiger, aber auch unterschiedlicher und spezifischer Kompetenzen bedarf, ist die Adressatenperspektive auch hier wieder - nach dem Kriterium der Wichtigkeit von Kompetenzen - ins Zentrum zu stellen, denn der Adressat als Person ist das (einzige!) Referenzkriterium, an dem sich alle (akteurs- und adressatenbezogenen) Kompetenzen zu orientieren haben. Schließlich dient die Soziale Arbeit (und ihre Kompetenz) dem Adressaten - und nicht umgekehrt!
bens-Kompetenzen) erweitert, ergänzt, spezifiziert und flankiert durch eine Reihe an Residualkompetenzen, mit Hilfe derer schwierige Lebenslagen, kritische Lebensereignisse und belastende Krisensituationen adäquat bewältigt werden können.

Diejenigen Kompetenzen, die das Zentrum der Kompetenz für Soziale Arbeit somit ausmachen, sind offensichtlich eine »Lebensführungskompetenz « (vgl. Volz 2009) und eine »Lebensbewältigungskompetenz « (vgl. Böhnisch 2005). Unschwer zu erraten, dass es sich dabei nicht primär um berufsorientierte Funktionskompetenzen von Akteuren im sozialen Feld handelt, sondern um Kompetenzen für das Leben von Adressaten, die - wie es auch der Capabilities-Ansatz postuliert - ein erfülltes, gedeihliches, menschliches und glückliches Leben führen wollen (vgl. Otto \& Ziegler 2010).

Aus diesem Grund sind auch sämtliche Kompetenzen aller in der Sozialen Arbeit Tätigen daran zu orientieren, eine umfassende Hilfe zum Erwerb der zentralen Kompetenzbereiche für die Adressaten anbieten zu können. Erst durch den Fokus auf die Lebensführungs- und die Lebensbewältigungskompetenz erhält die Soziale Arbeit nicht nur ihre Legitimation für ihr professionelles Handeln, ein begründbares, klares Ausbildungsprofil und einen allgemein anzuerkennenden Forschungsgegenstand, sondern sie wird dadurch auch professionsethischen Anforderungen gerecht, mit denen dem Anspruch einer humanistischen Grundorientierung im Sinne einer Hilfe zur Mündigkeit, Selbstbestimmung und selbsttätigen Lebensverwirklichung genüge getan wird (vgl. Mührel 2010).

\section{Die Stimme des Adressaten ... und die Gehörlosigkeit der Sozialen Arbeit}

Im Rahmen aller professioneller Hilfen, die Soziale Arbeit leisten kann, geht es daher nicht (nur) um alltägliche oder berufliche, sondern vor allem um existenzsichernde Kompetenzen, die aus der Perspektive des Adressatennutzens und des Akteur-Potentials zueinander vermittelt werden müssen. Denn: Für moderne Professionen ist die "Kategorie der Vermittlung « ihrer Sachthematiken und das Verstehen dieser Sachthematiken von den Klienten zentral (vgl. Ferchhoff 2009). Wenn dies jedoch nicht intendiert ist und die Probleme von den Professionellen für die Adressaten technologisch - und nicht mit den Adressaten dialogisch - gelöst werden, kann keine Strukturänderung, kein Strukturaufbau und kein Identitätserwerb der Personen gelingen.

Die professionelle Soziale Arbeit muss die Autonomie der Lebenspraxis ihrer Adressaten wertschätzen und die Subjekte selbststeuernd in den Lebensverhältnissen im Sinne ihrer Lebensführungs- und Lebensbewältigungs-Möglichkeiten zum selbstverantwortlichen Handeln in ihrer biographischen Entwicklung unterstützen (vgl. Lutz 2008). Darum hat es der Sozialen Arbeit, wenn sie denn überhaupt nach einer Klärung etwaiger Kompetenzen fragt, zuvörderst um die Kompetenzbereiche ihrer Adressaten und - damit um die Existenzialien des Lebens zu gehen. Und gerade auch deshalb lohnt sich der biographiebezogene Blick auf die Adressaten und deren Leben, Lebenslagen und Lebensprobleme, die nur durch ihre erzählbaren Geschichten deutlich werden. Denn jede Person hat ihre konkrete Geschichte, ihre Identität und ihre emotionale Verfassung und sie orientiert ihr Leben an Werten, Haltungen, Einstellungen und Erfahrungen, die seitens der professionellen Sozialen Arbeit auch gehört und erhört werden wollen (vgl. Birgmeier 2009)! 
In den Geschichten der Menschen, in ihrer Historizität wird der Bedarf an notwendigen Kompetenzen erst sichtbar; erst dort erhält eine Soziale Arbeit ihren eigentlichen Handlungsauftrag, in dem sie nach dem einzelnen Menschen in seiner historisch gewachsenen, lebensweltlichen Ganzheit fragt und nach dem, warum Adressaten der Sozialen Arbeit in ihren Selbstschöpfungs-, Sinngebungs- und Handlungsvollzügen Probleme in Bezug auf ihre Lebensführung und Lebensbewältigung haben.

Um überhaupt Antworten auf dieses Warum zu erhalten, ist - selbstredend zunächst einmal die Stimme des Adressaten (Bitzan et al. 2006) zu hören und der unmittelbare Kontakt mit dem Adressaten herzustellen. Erst dann kann die Soziale Arbeit aus einem Wissen schöpfen, das aus der "Innenperspektive « oder anders formuliert: aus dem tatsächlichen Leben und Erleben ihrer Klienten entstammt und könnte es verhindern, ihren Adressaten standardisierte Lösungen oder Modelle »überzustülpen«, die nicht zu deren Erwartungen, Erfahrungen und Lebensweisen passen.

\section{Ein Fazit}

Kompetenz hat immer etwas zu tun mit Zuständigkeit, mit Bereitschaft und mit Fähigkeit. Jemand, der sich mit dem Adjektiv »kompetent « zu schmücken trachtet, ist demnach auch für etwas zuständig, zu etwas bereit und zu etwas fähig. Doch wofür? Für alles, für manches oder für nichts?

Die Soziale Arbeit kann und darf sich nicht für alles zuständig, bereit und fähig fühlen. Vielmehr hat sie das, was schon im Sozialhilfegesetzbuch formuliert wurde, zu beachten. In diesem Gesetz wird eine dezidiert adressatenbezogene Leitperspektive proklamiert, das den sich selbst bestimmenden, mündigen und selbstverantwortlich handeln wollenden Klienten fokussiert, der das durch die Profession Soziale Arbeit initiierbare Geschehen - freilich im Rahmen eigener

\section{Literatur}

Birgmeier, B. (2009): Odo Marquards philosophisch-anthropologische Implikationen zum »Menschlichen«. In: Mührel, E. (Hg.): Zum Personenverständnis in der Sozialen Arbeit und der Pädagogik. Blaue Eule: Essen, 19-42.

Bitzan, M./Bolay, E./Thiersch, H. (2006) (Hg.): Die Stimme des Adressaten. Juventa: Weinheim.

Böhnisch, L. (2005): Sozialpädagogik der Lebensalter. Juventa: Weinheim.

Effinger, H. (2009): Begriffe, Bahnsteige und Gebietsansprüche bei der Erklärung und Bearbeitung sozialer Probleme. In: Birgmeier, B./Mührel, E. (Hg.): Die Sozialarbeitswissenschaft und ihre Theorie(n). VS: Wiesbaden, 52-67.

Ferchhoff, W. (2009): Prozesse der Professionalisierung in historischer und gegenwartsorientierter Perspektive. In: Birgmeier, B./Mührel, E. (Hg.): Die Sozialarbeitswissenschaft und ihre Theorie(n). VS: Wiesbaden, 69-84.

Lutz, R. (2008): Perspektiven der Sozialen Arbeit. In: Aus Politik und Zeitgeschichte $1 / 2008,1-10$.

Marquard, O. (2005): Inkompetenzkompensationskompetenz? In: ders. (Hg.): Abschied vom Prinzipiellen. Reclam: Stuttgart, 23-38.

Mührel, E. (2010): Entfremdete Sozialräume und desorientierte Lebenswelten. In: Birgmeier, B./Mührel, E./Schmidt, H.-L. (Hg.): Sozialpädagogik und Integration. Blaue Eule: Essen, 77-91.

Otto, H.-U./Ziegler, H. (2010) (Hg.): Capabilities - Handlungsbefähigung und Verwirklichungschancen in der Erziehungswissenschaft. VS: Wiesbaden.

Schmidt, H.-L. (2010): Integration durch Partizipation. In: Birgmeier, B./Mührel, E./Schmidt, H.-L. (Hg.): Sozialpädagogik und Integration. Blaue Eule: Essen, 197220.

Volz, F.R. (2009): »In aller Freundschaft « - Thesen zur Personwerdung und Vermögensbildung. In: Mührel, E./Birgmeier, B. (Hg.): Theorien der Sozialpädagogik ein Theorie-Dilemma? VS: Wiesbaden, 287-305.

Möglichkeiten - wesentlich mitbestimmt.

Ganz offensichtlich ist daher - insbesondere dann, wenn wir über Kompetenzen sprechen wollen - nicht nur der Adressat ein Experte in eigener Sache, sondern der Begriff der Partizipation ein Zentralbegriff für eine an Kompetenzen orientierte Soziale Arbeit. Im Begriff der Partizipation nämlich wird das deutlich, was dieser Terminus in seiner lateinischen Wurzel eindeutig beinhaltet: Partem capere - bedeutet nämlich: seinen Teil ergreifen, nehmen und teilnehmen (vgl. Schmidt 2010).

Es ist längst an der Zeit, dass den Adressaten ihr Anteil an der Sozialen Arbeit (wieder) zurückgegeben wird - in dem man sie ins Zentrum der Disziplin, der Profession und der Ausbildung in Sozialer Arbeit rückt und die Kompetenzen in diesen drei Bereichen an den zentralen Kompetenzen für eine gelingende und gute Lebensführung und Lebensbewältigung orientiert.

Erst dann dürfen wir (wieder) behaupten, es ginge uns tatsächlich um den Menschen ... 
"Wenn viele zusammen nichts tun,

so ist das noch lange keine Kooperation."

Wolfgang Tilgner, deutscher Schriftsteller (geb. 1932)

"Kein Plan überlebt den Kontakt mit dem Feind. "

Sunzi, chinesischer General (um 500 v. Chr.)

"Wer davon lebt, einen Feind zu bekämpfen,

hat ein Interesse daran, dass er am Leben bleibt."

Friedrich Nietzsche, deutscher Philosoph (1844-1900)

"Ich weiß viel zu wenig, als dass ich inkompetent sein könnte." Woody Allen, amerikanischer Regisseur und Schriftsteller (geb. 1935)

"Nicht die Ressourcen als solche sind wesentlich,

sondern wesentlich ist deren Nutzung für

produktive Zwecke, für die Schaffung von Werten."

Prof. Dr. Fredmund Malik, österreichischer Managementberater (geb. 1944)

"Freiheit haust in jenen Gebieten, die zwar organisiert, doch nicht zur

Organisation zu zählen sind."

Ernst Jünger, deutscher Schriftsteller und Philosoph (1895-1998)

„Organisationen halten Leute beschäftigt, unterhalten sie bisweilen, vermitteln ihnen eine Vielfalt von Erfahrungen,

halten sie von den Straßen fern, liefern Vorwände

für Geschichtenerzählen und ermöglichen

Sozialisation. Sonst haben sie nichts anzubieten."

Karl E. Weick, amerikanischer Soziologe (geb. 1936) 